\title{
Effects of Long-Term Bottle Storage on Red and Rosé Wines Sealed with Different Types of Closures
}

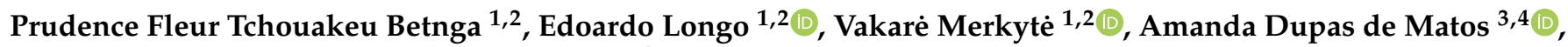 \\ Fabrizio Rossetti ${ }^{5}$ and Emanuele Boselli $1,2, *$ (D) \\ 1 Faculty of Science and Technology, Free University of Bozen-Bolzano, Piazza Università 1, \\ 39100 Bolzano, Italy; prudencefleur.tchouakeubetnga@natec.unibz.it (P.F.T.B.); edoardo.longo@unibz.it (E.L.); \\ vakare.merkyte2@unibz.it (V.M.) \\ 2 Oenolab, NOI Techpark Alto Adige/Südtirol, Via A. Volta 13B, 39100 Bolzano, Italy \\ 3 Food Experience and Sensory Testing (Feast) Lab, Massey University, Palmerston North 4410, New Zealand; \\ a.dupasdematos@massey.ac.nz \\ 4 Riddet Institute, Massey University, Palmerston North 4410, New Zealand \\ 5 Mérieux NutriSciences, Via Marradi 41, 59100 Prato, Italy; fabrizio.rossetti@mxns.com \\ * Correspondence: emanuele.boselli@unibz.it
}

\section{check for} updates

Citation: Tchouakeu Betnga, P.F.; Longo, E.; Merkytè, V.; Dupas de Matos, A.; Rossetti, F.; Boselli, E. Effects of Long-Term Bottle Storage on Red and Rosé Wines Sealed with Different Types of Closures. Foods 2021, 10, 2918. https://doi.org/ $10.3390 /$ foods 10122918

\section{Academic Editors: José}

Sousa Câmara, José António Couto Teixeira and Rosa M. Perestrelo

Received: 9 October 2021

Accepted: 22 November 2021

Published: 25 November 2021

Publisher's Note: MDPI stays neutral with regard to jurisdictional claims in published maps and institutional affiliations.

Copyright: (c) 2021 by the authors. Licensee MDPI, Basel, Switzerland. This article is an open access article distributed under the terms and conditions of the Creative Commons Attribution (CC BY) license (https:// creativecommons.org/licenses/by/ $4.0 /$ )

\begin{abstract}
Volatile and non-volatile chemical profiles, free and total $\mathrm{SO}_{2}$ and dissolved oxygen content were studied in three red (Merlot, Lagrein red, St. Magdalener) and one rosé (Lagrein rosé) wine after 30 months of storage in bottles. Each wine was sealed with closures made of a 'blend' (B) of natural cork microgranules and polymers without glue and was compared with wines closed with other types of corks (C; a technical cork $1+1$, or an agglomerated natural cork or a natural one-piece cork). Glutathionyl caftaric acid (GRP) was inversely correlated with total $\mathrm{SO}_{2}$ content and was higher in all three red wines closed with B compared to $C$, whereas epicatechin was higher in three wines closed with C compared to B. Three volatile compounds formed by fermentation (ethyl butanoate, isoamyl lactate, and octanol) were inversely correlated with both free and total $\mathrm{SO}_{2}$. In terms of their volatile profiles, ethyl octanoate and 2,3-butanediol were significantly higher in the Lagrein red wines closed with C closures, whereas no significant difference was observed in Merlot, Lagrein rosé and St. Magdalener wines. Small differences in some phenolic compounds due to the type of closure were found: GRP, syringic acid, (+)-catechin, and (-)-epicatechin differentiated the Merlot wines closed with $B$ from the $C$ closures. Protocatechuic acid and GRP levels differentiated the Lagrein red wines according to their closure type, whereas only (-)-epicatechin differentiated the Lagrein rosé wines. GRP, caffeic acid, (-)-epicatechin, and anthocyanin content differentiated the St. Magdalener wines according to their closure type. Even though St. Magdalener and Lagrein rosé closed with C could be distinguished from those closed with B by using the (sensory) triangle test $(\alpha=0.05)$, these differences appeared to be relative as it did not include all the wines in a systematic manner.
\end{abstract}

Keywords: closures; dissolved oxygen content; sulfur dioxide; phenolic compounds; volatile profile; bottle storage; wine ageing

\section{Introduction}

Some wines are intended to be stored for a short period of time before their consumption; in contrast, many premium wines are aged for several years or even decades [1]. Over the period of a bottle's ageing, a wine gradually alters to reach maturity, at which point its organoleptic quality is maximally expressed; then, this period is followed by a phase of decline in quality [2]. The maturation of bottled wine is strongly linked to its composition at the time of bottling. In addition, storage conditions such as temperature and light exposure in the cellar are also relevant to the maturation of wine [3], but not all of them can influence the different aspects of wine quality in the same way (for example, aroma and color profiles). Wine ageing in bottles can be impacted by the quality of the closure 
used [4-7], which can drastically affect the commercial quality of the product, consumer preferences and, in general, the reputation of the winery. For example, a natural one-piece cork closure of low quality may be too permeable to air or may contract due to dehydration (due to a very low environmental relative humidity), leading to breakage of the closure, or to wine oxidation [8-12]. It is well known that the aroma of wine tends to develop because of numerous reactions. Wine can benefit from slight exposure to oxygen, as this allows color stabilization, favors the reduction of astringency and the development of specific aroma components [13]. This is particularly important for red wines, as they contain high levels of phenolic compounds, i.e., the main reactants with oxygen in wine [14]. Controlled oxidation can also prove beneficial to white wines, such as Riesling, which develops a golden (rather than brown) color and an aroma typical of aged wine [12,15,16]. A gradual maturation can also take place in an environment, such as in a bottle, which has a very low oxygen content $[17,18]$. In most scientific works, the maturation of wines exposed to oxygen after bottling has been evaluated, focusing on the relationship between the closure and wine exposure to oxygen $[10,18,19]$. Indeed, a high oxygen content or a low free sulfite content can lead to oxidative deterioration and consequently will result in some off-odors and/or off-flavors in the bottled wine [20,21].

Numerous studies have described the influence of closures during the maturation of wines after bottling, with the aim of establishing the optimal conditions for improving their organoleptic quality $[5,19,22]$. Oxygen exposure is the most important factor, along with the type of closure-although several other factors are reported as determinants during the post-bottling phase, such as light exposure [23,24], temperature fluctuations [25,26], vibrations [27,28], and humidity.

The effect of different closure materials on wine development has also been studied [6,28-32]. Godden et al. [29] evaluated the performance of different closures (natural cork, technical cork, screw cap, and synthetic closures) on bottled wines after 20 months of storage. The results showed that the highest retention of sulfites and ascorbic acid, as well as the slowest rate of browning, were recorded in wine bottles closed with screw caps. The direct interactions of the closure with the wine has been an important subject of research. González-Adrados et al. [6] conducted a study using two different closure materials (natural cork and $1+1$ technical cork) with treated and non-treated surfaces to evaluate the interactions between the closure and the wine. Their results showed that most of the overall migration of non-volatile compounds from the closure to the wine was due to the natural components of the cork. Closure type (natural cork or $1+1$ technical cork closures) and contact time accounted for the greatest variability, while surface treatment increased overall migration and decreased liquid absorption.

The first part of the present research [33] provided details on the maturation of four wines (one rosé and three reds from Alto Adige/South Tyrol, Italy) during up to 12 months of storage in bottles sealed with different closures.

In this study, the profiles of volatile and non-volatile compounds, free and total $\mathrm{SO}_{2}$ content, and dissolved oxygen content of the same wines were analyzed after 30 months of storage in bottles, with the goal of evaluating how quality was affected by the different types of closures over a much longer period of time than previously studied. In addition, a sensory discrimination test was applied to assess the differences between samples closed with two different closures ('conventional' vs 'blend'). The results obtained broadened the knowledge of closure-wine interactions after a long period of storage in the bottle.

\section{Materials and Methods}

\subsection{Wines and Closures}

Four wines (Merlot, Lagrein red, Lagrein rosé and St. Magdalener) were provided by a local winery (Kellerei Bozen, Bolzano, Italy). The grapes were harvested in 2016 from vineyards located in the same area. Each of the four types of wine was made using the winery's standard winemaking protocol for that particular wine; the wines were then bottled in the following year. The only difference between the bottles of each different 
wine was the type of closure used. The different closures were: a 'blend' cork (a sanitized micro-granule blend of natural cork with polymers without the addition of glue to the mix) and 'conventional' closures (an agglomerated natural cork, a natural one-piece cork and a technical cork $1+1$ ) from different suppliers, as reported in Table 1 . A total number of $16(0.75 \mathrm{~L})$ bottles with 4 bottles of each type of wine were sampled. All the bottles were stored horizontally at a cellar temperature (about $16{ }^{\circ} \mathrm{C}, 50 \%$ relative humidity) for most of the storage time. The bottles were opened after 30 months of storage to characterize their volatile and phenolic profiles, as well as their dissolved oxygen, free and total $\mathrm{SO}_{2}$ content and to perform the triangle test. At the time of analysis, all the bottles were stored in the laboratory for a short time under a constant controlled temperature $\left(23^{\circ} \mathrm{C}\right)$. A total of four bottles of each type of wine (two replicate samples for each type of closure) were analyzed.

Table 1. The three different 'conventional' closures used to close the wine bottles which were compared with a control closure ('blend' cork).

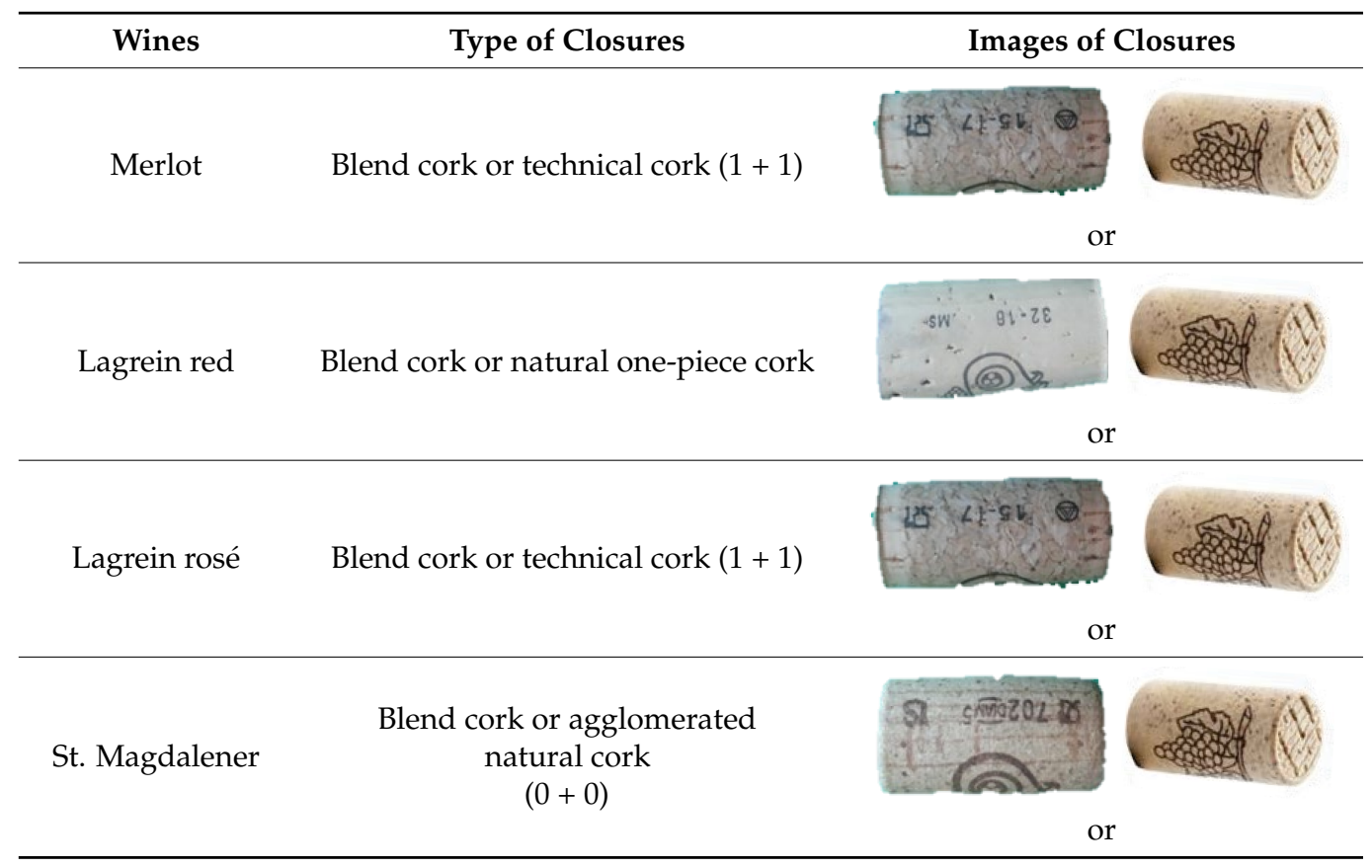

\subsection{Analysis of Volatile Compounds}

Volatile compounds were sampled by head-space solid phase microextraction (HSSPME), according to the operating parameters described by Rossetti et al. [33]. Then, they were determined by gas chromatography-mass spectrometry (GC-MS) with slight modifications. Briefly, $1 \mathrm{~g} \mathrm{NaCl}$ was introduced into a vial $(20 \mathrm{~mL})$ containing $10 \mathrm{~mL}$ of wine; the vial was then closed with a screw cap fitted with a perforable elastomeric septum and was placed in a heating bath at $40{ }^{\circ} \mathrm{C}$ for $10 \mathrm{~min}$. The vial was continuously stirred at $270 \mathrm{rpm}$ to reach equilibrium. Afterwards, a SPME fiber (DVB/CAR/PDMS, 50/30 $\mu \mathrm{m}$, $1 \mathrm{~cm}$, Agilent, Santa Clara, CA, USA) was introduced into the sample headspace for $20 \mathrm{~min}$ under the same heating and mixing conditions.

For GC-MS analysis, a 7890A gas chromatograph was coupled to a 5975 mass spectrometer both from Agilent (Wilmington, DE, USA). Volatile compounds were thermally desorbed at $240{ }^{\circ} \mathrm{C}$ for $3.5 \mathrm{~min}$ and analyzed using a HP-5MS capillary column $(0.25 \mu \mathrm{m} / 0.25 \mathrm{~mm} / 30 \mathrm{~m}$; Agilent, Wilmington, USA). The injector was set to splitless mode and the temperature program of the GC oven was as follows: $40{ }^{\circ} \mathrm{C}$ for $2.5 \mathrm{~min}$, then increased to $180{ }^{\circ} \mathrm{C}$ at a rate of $3{ }^{\circ} \mathrm{C} / \mathrm{min}$ and finally up to $230^{\circ} \mathrm{C}$ at $10^{\circ} \mathrm{C} / \mathrm{min}$. Helium, as the carrier gas, was set to a constant flow mode $(0.7 \mathrm{~mL} / \mathrm{min})$. The electron ionization was set at an energy of $70 \mathrm{eV}$ and the ion source temperature was set at $230^{\circ} \mathrm{C}$. The mass 
range of the detector was $34-360 \mathrm{~m} / \mathrm{z}$; the quadrupole temperature was $150{ }^{\circ} \mathrm{C}$, and the acquisition rate was $1 \mathrm{scan} / \mathrm{sec}$.

\subsection{HPLC Analysis}

The analysis of the phenolic compounds was performed according to Longo et al. [34] and Dupas de Matos et al. [35]. Briefly, the separation was carried out on an ODS column (Eurosphere II, C18 stationary phase, $250 \times 4.6 \mathrm{~mm}, 5 \mu \mathrm{m}$ produce by Knauer) using a Nexera X2 UHPLC (Shimadzu, Milan, Italy) coupled with a UV-Vis diode array detector (Shimadzu). The HPLC flow rate was $0.7 \mathrm{~mL} \mathrm{~min}^{-1}$. The HPLC mobile phase consisted of solvent A ( $0.1 \%$ formic acid in degassed milliQ water) and solvent $\mathrm{B}(0.1 \%$ formic acid in acetonitrile). The gradient method was the following: $0-2.5 \mathrm{~min}, 99 \% \mathrm{~A} ; 2.5-50 \mathrm{~min}$ $99-75 \%$ A; 50-51 min 75-1\% A; 51-55 min 1\% A; 55-56 min 1-99\% A; 56-60 min 99\% A.

Phenolic compounds were identified by comparing their chromatographic retention times and UV-Vis spectra with those of pure standard compounds. For this purpose, solutions of standard compounds were injected into the HPLC system. Peaks of different chromatograms were aligned manually. Then, the peak areas of the analytes were integrated using the LabSolutions System software by Shimadzu (Milan, Italy). Calibration curves of pure standard substances ( $\geq 98 \%$, Sigma Aldrich, Milan, Italy) were established through the DAD and were used to quantify the concentrations of phenolic compounds. When reference compounds were not available, a calibration with structurally related standard substances was used (gallic acid for protocatechuic acid and syringic acid; caffeic acid for caftaric acid and glutathionyl caftaric acid (GRP); (+)-catechin for (-)-epicatechin) ( $\geq 98 \%$, Sigma Aldrich, Milan, Italy). The peak areas were integrated to obtain the concentrations of the identified compounds.

The statistical elaboration of the HPLC data was performed using XLStat (version 2019.2.2.59417, Addinsoft, Paris, France).

\subsection{Determination of Dissolved $\mathrm{O}_{2}$ and $\mathrm{SO}_{2}$}

A non-invasive optical sensor (L. sensor-700.O2) from an FT system (Alseno, Italy) was used to measure the dissolved oxygen content of wines. This is an IR laser type analyser with a measurement range of 3-21\% $\mathrm{O}_{2} \pm 0.3 \%$ and an accuracy of $\pm 0.2 \% \mathrm{O}_{2}$ concentration. Free and total $\mathrm{SO}_{2}$ content of wines were analyzed using the Miura One multiparametric analyzer from Exacta + Optech Labcenter (San Prospero, Italy).

\subsection{Sensory Discrimination Test}

A triangle test according to the ISO 4120:2007 method was chosen to evaluate the differences between wines sealed with a 'conventional' closure and the 'blend' closure.

The sensory panel was formed by 11 assessors ( 8 males and 3 females, $23 \pm 4$ years old) who were recruited from among the enology students and technical staff of the Faculty of Science and Technology.

The panelists received 4 sets of 3 wines and were asked to evaluate one set at a time, selecting the odd sample out of each set. Fifty $\mathrm{mL}$ of wine were poured into ISO glasses; the glasses were labeled with random three-digit codes. The wine glasses were then presented to the panelists (at a temperature of $18^{\circ} \mathrm{C}$ ) in a random order, as per the standard methodology for the triangle test. The sensitivity parameters of the test were set at $\alpha=0.5, \beta=0.20$ and $p d=50 \%$. Two sessions were organized due to the relatively low number of assessors.

\subsection{Multivariate Data Analysis}

Multivariate statistical processing of the results was performed to explore the structure of the experimental data using XLStat software (version 2019.2.2.59417, Addinsoft, Paris, France). An alpha value of 0.05 was chosen to determine statistical significance, unless otherwise stated. Regarding the chemical profiles, statistical treatment was performed on the relative abundance of the analyte areas. One-way ANOVA was applied to the data, 
using closure type as the independent variable, followed by Tukey's test for post-hoc mean separation. Principal component analysis (PCA) was applied, averaging the same-bottle data (technical repetitions).

\section{Results}

\subsection{Profile of Volatile Compounds}

A total number of 30 volatile compounds were identified in the wines during the 30 months [33] of bottle storage.

Table 2 shows the volatile compounds identified in the four wine samples over 12 months of storage compared to those identified after 30 months. A total of 30 volatile compounds were identified; however, a considerable loss of some of these compounds was observed after long-term storage ( 30 months). Thirteen compounds (4-ethylbenzoic acid, 2-butyl ester; 1-heptanol; 1-octen-3-ol; hexyl acetate; limonene; 2-ethyl hexanol; methyl benzaldehyde; ethyl benzaldehyde; methyl salicylate; benzenacetic acid, ethyl ester; 2-phenylethylacetate; ethyl dodecanoate; ethyl hexadecanoate) which were initially present in all the wine samples up to twelve months, were not detected at thirty months. On the other hand, four volatile compounds (3-methyl-1-butanol; 2,3-butanediol; 2-hydroxyethyl propanoate; isoamyl lactate) identified at thirty months were not detected in the first twelve months of storage. An interpretation of this observation could be that during storage, some long-chained fresh fruity ethyl esters were hydrolysed to form alcohols and that some esters appeared after 30 months storage-probably due to the condensation of higher alcohols (e.g., isoamyl alcohol) with organic acids formed after fermentation. Additionally, some C7 and C8 higher alcohols and aldehydes disappeared-probably due to oxidation.

Table 2. Volatile compounds identified during 30 months of storage listed according to their elution order. LRI $=$ Linear retention index.

\begin{tabular}{|c|c|c|c|c|c|c|c|}
\hline & $\begin{array}{c}\text { Volatile } \\
\text { Compounds }\end{array}$ & $\begin{array}{l}\text { Over } 12 \text { Months } \\
\text { Storage as } \\
\text { in [33] }\end{array}$ & $\begin{array}{l}\text { At a 30-Month } \\
\text { Storage }\end{array}$ & $\begin{array}{c}\text { LRI } \\
\text { (Ref./NIST) }\end{array}$ & $\begin{array}{l}\text { LRI (Mea- } \\
\text { sured) }\end{array}$ & $\begin{array}{l}\text { Base Peak } \\
(\mathrm{m} / \mathrm{z})\end{array}$ & $\begin{array}{l}\text { Fragmentation } \\
\text { Pattern }(\mathrm{m} / \mathrm{z})\end{array}$ \\
\hline 1 & Acetic acid & $\sqrt{ }$ & $\sqrt{ }$ & $599[36]$ & / & 43 & $43 ; 45 ; 60$ \\
\hline 2 & $\begin{array}{l}\text { 3-methyl-1- } \\
\text { butanol }\end{array}$ & $X$ & $\sqrt{ }$ & 732 [37] & 705 & 55 & $42 ; 55 ; 70$ \\
\hline 3 & 2,3-butanediol & $X$ & $\sqrt{ }$ & $782[38]$ & 782 & 45 & $45 ; 57$ \\
\hline 4 & Ethyl butanoate & $\sqrt{ }$ & $\sqrt{ }$ & $803[36]$ & 772 & 71 & $43 ; 71 ; 88$ \\
\hline 5 & $\begin{array}{l}\text { 2-hydroxyethyl } \\
\text { propanoate }\end{array}$ & $x$ & $\sqrt{ }$ & 793 [39] & 792 & 57 & $45 ; 57 ; 75 ; 87 ; 88$ \\
\hline 6 & $\begin{array}{c}\text { Ethyl ester of } \\
\text { 2-methylbutanoic } \\
\text { acid }\end{array}$ & $\sqrt{ }$ & $\sqrt{ }$ & $846[36]$ & 824 & 57 & $57 ; 74 ; 85 ; 102$ \\
\hline 7 & $\begin{array}{c}\text { Ethyl ester of } \\
\text { 3-methylbutanoic } \\
\text { acid }\end{array}$ & $\sqrt{ }$ & $\sqrt{ }$ & 859 [40] & 827 & 88 & $41 ; 57 ; 70 ; 88$ \\
\hline 8 & 1-Hexanol & $\sqrt{ }$ & $\sqrt{ }$ & $865[41]$ & 843 & 56 & $56 ; 69 ; 84$ \\
\hline 9 & Isopentyl acetate & $\sqrt{ }$ & $\sqrt{ }$ & $876[41]$ & 850 & 43 & $43 ; 55 ; 70$ \\
\hline 10 & $\begin{array}{c}\text { 2-butylester of } \\
\text { 4-ethylbenzoic acid }\end{array}$ & $\sqrt{ }$ & $x$ & / & / & 133 & $105 ; 151$ \\
\hline 11 & 1-Heptanol & $\sqrt{ }$ & $X$ & $969[41]$ & / & 70 & $\begin{array}{c}41 ; 42 ; 43 ; 55 ; 56 ; \\
70\end{array}$ \\
\hline 12 & 1-Octen-3-ol & $\sqrt{ }$ & $x$ & $980[36]$ & / & 57 & $43 ; 55 ; 57 ; 72$ \\
\hline 13 & Ethyl hexanoate & $\sqrt{ }$ & $\sqrt{ }$ & 999 [41] & 975 & 88 & $43 ; 60 ; 70 ; 88 ; 99$ \\
\hline 14 & Hexyl acetate & $\sqrt{ }$ & $X$ & $1011[41]$ & / & 43 & $\begin{array}{c}43 ; 55 ; 56 ; 61 ; 69 ; \\
84\end{array}$ \\
\hline 15 & Limonene & $\sqrt{ }$ & $x$ & 1020 [41] & / & 68 & $67 ; 68 ; 93$ \\
\hline 16 & 2-Ethyl hexanol & $\sqrt{ }$ & $x$ & $1028[42]$ & / & 57 & $41 ; 43 ; 57$ \\
\hline 17 & Isoamyl lactate & $x$ & $\sqrt{ }$ & $1047[43]$ & 1042 & 45 & $43 ; 45 ; 55 ; 70$ \\
\hline
\end{tabular}


Table 2. Cont.

\begin{tabular}{|c|c|c|c|c|c|c|c|}
\hline & $\begin{array}{l}\text { Volatile } \\
\text { Compounds }\end{array}$ & $\begin{array}{l}\text { Over } 12 \text { Months } \\
\text { Storage as } \\
\text { in [33] }\end{array}$ & $\begin{array}{l}\text { At a 30-Month } \\
\text { Storage }\end{array}$ & $\begin{array}{c}\text { LRI } \\
\text { (Ref./NIST) }\end{array}$ & $\begin{array}{l}\text { LRI (Mea- } \\
\text { sured) }\end{array}$ & $\begin{array}{l}\text { Base Peak } \\
\quad(\mathrm{m} / \mathrm{z})\end{array}$ & $\begin{array}{l}\text { Fragmentation } \\
\text { Pattern }(\mathrm{m} / \mathrm{z})\end{array}$ \\
\hline 18 & $\begin{array}{c}\text { 4-Methyl } \\
\text { benzaldehyde }\end{array}$ & $\sqrt{ }$ & $x$ & 1076 [44] & / & 91 & $65 ; 91 ; 119 ; 120$ \\
\hline 19 & Octanol & $\sqrt{ }$ & $\sqrt{ }$ & $1070[41]$ & 1055 & 56 & $\begin{array}{c}41 ; 42 ; 43 ; 55 ; 56 \\
69 ; 70 ; 84\end{array}$ \\
\hline 20 & $\begin{array}{c}4- \\
\text { Ethylbenzaldehyde }\end{array}$ & $\sqrt{ }$ & $x$ & 1163 [45] & / & 134 & $91 ; 105 ; 133 ; 134$ \\
\hline 21 & 2-Phenylethanol & $\sqrt{ }$ & $\sqrt{ }$ & 1112 [36] & 1084 & 91 & $65 ; 91 ; 122$ \\
\hline 22 & Octanoic acid & $\sqrt{ }$ & $\sqrt{ }$ & $1180[46]$ & 1152 & 60 & 60; 73; 101 \\
\hline 23 & Diethyl succinate & $\sqrt{ }$ & $\sqrt{ }$ & 1179 [47] & 1155 & 101 & $\begin{array}{c}45 ; 55 ; 73 ; 101 ; \\
129\end{array}$ \\
\hline 24 & Methyl salicylate & $\sqrt{ }$ & $x$ & 1192 [41] & / & 120 & $92 ; 120 ; 121 ; 152$ \\
\hline 25 & Ethyl octanoate & $\sqrt{ }$ & $\sqrt{ }$ & $1194[48]$ & 1169 & 88 & $\begin{array}{c}41 ; 57 ; 73 ; 88 ; 101 ; \\
115 ; 127\end{array}$ \\
\hline 26 & $\begin{array}{c}\text { Benzeneacetic acid, } \\
\text { ethyl ester }\end{array}$ & $\sqrt{ }$ & $X$ & $1243[41]$ & / & 91 & $164 ; 91 ; 65$ \\
\hline 27 & $\begin{array}{c}2- \\
\text { Phenylethylacetate }\end{array}$ & $\sqrt{ }$ & $X$ & $1255[41]$ & / & 104 & $43 ; 91 ; 104$ \\
\hline 28 & Ethyl decanoate & $\sqrt{ }$ & $\sqrt{ }$ & 1403 [49] & 1361 & 88 & $73 ; 88 ; 101 ; 155$ \\
\hline 29 & Ethyl dodecanoate & $\sqrt{ }$ & $x$ & $1554[41]$ & / & 88 & $43 ; 73 ; 88 ; 101$ \\
\hline 30 & $\begin{array}{l}\text { Ethyl hexade- } \\
\text { canoate }\end{array}$ & $\sqrt{ }$ & $x$ & $1992[50]$ & / & 88 & $43 ; 88 ; 101$ \\
\hline
\end{tabular}

$\sqrt{ }$ : detected with internal area $>0.01 \%$; X: not detected (internal area $<0.01 \%$ ).

The differences in the volatile profile present in wines at 30 months storage were evaluated by one-way ANOVA, taking the closures as the independent factor (Table 3). Four individual one-way ANOVAs were carried out for each wine to test the differences between the 'blend' closure and the 'conventional' one. As a result, only red Lagrein showed a significant difference for 2,3-butanediol (3) and ethyl octanoate (25), which were both higher in red Lagrein wines closed with 'conventional' closures than in those closed with the 'blend' closure. No significant difference was found in St. Magdalener, Merlot, nor Lagrein rosé closed with 'conventional' or 'blend' closures. The volatile compounds that contributed the most to the rosé Lagrein wines sealed with both 'conventional' and 'blend' closures after 30 months of storage were a diol, a higher alcohol, and 3 esters-which are claimed to give pungent and fruity/floral notes to wines-respectively [51,52]. These compounds were butanediol (3), 1-hexanol (8), ethyl hexanoate (13), ethyl octanoate (25), and ethyl decanoate (28).

The most volatile compounds, such as 3-methyl-1-butanol (2), ethyl butanoate (4), isoamyl lactate (17), octanol (19), and 2-phenylethyl alcohol (21), contributed to the profile of the St. Magdalener wines and are known as compounds that give fresh fruity and floral aromas - excluding 3-methyl-1-butanol and octanol, which give pungent and mushroom/musty notes, respectively [53].

It is also worth noting that octanoic acid (22) and diethyl succinate (23; two volatile compounds associated with fermentations and/or oxidation reactions [33]), and three esters: 2-hydroxyethyl propanoate (5), 2-methylethyl butanoate (6), and 3-methylethyl butanoate (7), distinguished the Merlot samples. 


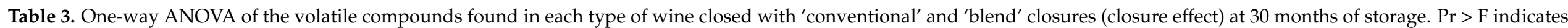
the $p$-value associated with the F statistic. The asterisk $\left(^{*}\right)$ means significantly different using Tukey's test $(\alpha<0.05)$. B and C, blend and conventional closure, respectively.

\begin{tabular}{|c|c|c|c|c|c|c|c|c|c|c|}
\hline \multicolumn{11}{|c|}{ Merlot } \\
\hline Closure & 3-Methyl-1-Butanol & \multicolumn{2}{|c|}{ 2-Hydroxyethyl Propanoate } & $\begin{array}{l}\text { 2-Methyl ethyl } \\
\text { Butanoate }\end{array}$ & $\begin{array}{l}\text { 3-Methyl ethyl } \\
\text { Butanoate }\end{array}$ & 1-Hexanol & $\begin{array}{l}\text { Isopenthyl } \\
\text { Acetate }\end{array}$ & $\begin{array}{c}\text { Ethyl } \\
\text { Hexanoate }\end{array}$ & $\begin{array}{l}\text { Isoamyl } \\
\text { Lactate }\end{array}$ & $\begin{array}{l}\text { 2-Phenyl } \\
\text { Ethanol }\end{array}$ \\
\hline C & 622245228 & \multicolumn{2}{|c|}{40645189} & 3806540 & 10057235 & 15511016 & 17334871 & 201290040 & 3860017 & 98766115 \\
\hline B & 820647919 & \multicolumn{2}{|c|}{46158129} & 5210750 & 13167523 & 20750259 & 39098612 & 192166261 & 4403087 & 175713260 \\
\hline $\operatorname{Pr}>F$ & 0.341 & \multicolumn{2}{|c|}{0.865} & 0.643 & 0.746 & 0.315 & 0.242 & 0.804 & 0.491 & 0.501 \\
\hline closure & Diethyl succinate & \multicolumn{2}{|c|}{ Octanoic acid } & $\begin{array}{c}\text { Ethyl } \\
\text { octanoate }\end{array}$ & $\begin{array}{c}\text { Ethyl } \\
\text { decanoate }\end{array}$ & & & & & \\
\hline C & 532233181 & \multirow{2}{*}{\multicolumn{2}{|c|}{$\begin{array}{c}128705315 \\
4616469\end{array}$}} & 430186222 & 16189337 & & & & & \\
\hline B & 249480078 & & & 510187046 & 31310515 & & & & & \\
\hline $\operatorname{Pr}>F$ & 0.423 & \multicolumn{2}{|c|}{0.415} & 0.862 & 0.568 & & & & & \\
\hline \multicolumn{11}{|c|}{ Lagrein red } \\
\hline Closure & 3-Methyl-1-butanol & 2,3-Butanediol & $\begin{array}{c}2- \\
\text { Hydroxyethyl } \\
\text { propanoate }\end{array}$ & 1-Hexanol & Isopentyl acetate & $\begin{array}{c}\text { Ethyl } \\
\text { hexanoate }\end{array}$ & $\begin{array}{c}2- \\
\text { Phenylethanol }\end{array}$ & $\begin{array}{c}\text { Diethyl } \\
\text { succinate }\end{array}$ & $\begin{array}{c}\text { Ethyl } \\
\text { octanoate }\end{array}$ & $\begin{array}{c}\text { Ethyl } \\
\text { decanoate }\end{array}$ \\
\hline $\mathrm{B}$ & 808285867 & 806359 & 37373403 & 22647330 & 60874527 & 189831224 & 129556887 & 237468679 & 535496801 & 42955096 \\
\hline $\operatorname{Pr}>F$ & 0.691 & $0.006^{*}$ & 0.166 & 0.809 & 0.214 & 0.137 & 0.179 & 0.223 & $0.010 *$ & 0.253 \\
\hline \multicolumn{11}{|c|}{ Lagrein rosé } \\
\hline Closure & 3-Methyl-1-butanol & 2,3-Butanediol & 1-Hexanol & $\begin{array}{c}\text { Isopentyl } \\
\text { acetate }\end{array}$ & Ethyl hexanoate & $\begin{array}{c}2- \\
\text { Phenylethanol }\end{array}$ & $\begin{array}{c}\text { Diethyl } \\
\text { succinate }\end{array}$ & $\begin{array}{c}\text { Ethyl } \\
\text { octanoate }\end{array}$ & \multicolumn{2}{|c|}{ Ethyl decanoate } \\
\hline C & 181321516 & 7388017 & 43588910 & 58215889 & 540146675 & 41387280 & 158422635 & 2441116143 & \multicolumn{2}{|c|}{4952211955} \\
\hline B & 297558079 & 47935843 & 45742412 & 63857184 & 509214725 & 70638784 & 210194281 & 1614962842 & \multicolumn{2}{|c|}{221721242} \\
\hline $\operatorname{Pr}>F$ & 0.118 & 0.487 & 0.550 & 0.792 & 0.436 & 0.359 & 0.331 & 0.065 & \multicolumn{2}{|c|}{0.131} \\
\hline
\end{tabular}


Table 3. Cont.

\begin{tabular}{|c|c|c|c|c|c|c|c|c|c|}
\hline \multicolumn{10}{|c|}{ St. Magdalener } \\
\hline Closure & Acetic acid & $\begin{array}{l}\text { 3-Methyl-1- } \\
\text { butanol }\end{array}$ & 2,3-Butanediol & $\begin{array}{c}\text { Ethyl } \\
\text { butanoate }\end{array}$ & $\begin{array}{l}\text { 2-Hydroxyethyl } \\
\text { propanoate }\end{array}$ & $\begin{array}{l}\text { 2-Methyl ethyl } \\
\text { butanoate }\end{array}$ & $\begin{array}{l}\text { 3-Methyl ethyl } \\
\text { butanoate }\end{array}$ & 1-Hexanol & Isopenthyl acetate \\
\hline $\mathrm{C}$ & 11505643 & 889501476 & 2914012 & 5280037 & 35636365 & 4369199 & 10416494 & 23082888 & 83015919 \\
\hline $\operatorname{Pr}>F$ & 0.414 & 0.533 & 0.342 & 0.751 & 0.574 & 0.382 & 0.835 & 0.234 & 0.701 \\
\hline Closure & Ethyl hexanoate & $\begin{array}{l}\text { Isoamyl } \\
\text { lactate }\end{array}$ & Octanol & $\begin{array}{l}\text { 2-Phenyl } \\
\text { ethanol }\end{array}$ & Diethyl succinate & Octanoic acid & $\begin{array}{c}\text { Ethyl } \\
\text { octanoate }\end{array}$ & $\begin{array}{c}\text { Ethyl } \\
\text { decanoate }\end{array}$ & \\
\hline C & 224036589 & 5092575 & 5513034 & 227373202 & 354489074 & 5627357 & 659384377 & 22794356 & \\
\hline B & 189855333 & 30786045 & 16732152 & 158471891 & 252616407 & 4325906 & 507270573 & 24993361 & \\
\hline $\operatorname{Pr}>F$ & 0.151 & 0.425 & 0.419 & 0.223 & 0.129 & 0.789 & 0.143 & 0.825 & \\
\hline
\end{tabular}


As for the red Lagrein wines, two volatile compounds-acetic acid (1) and 2-phenylethyl alcohol (21) — distinguished the samples.

In summary, there was no differentiation between bottles closed with the two different types of closures after 30 months except for one case-the Lagrein red wine, as highlighted above by the one-way ANOVA. The ANOVA showed no statistically significant differences in terms of the effects of the closure for the Merlot, Lagrein rosé or St. Magdalener.

\subsection{Non-Volatile Compounds}

Gallic acid, protocatechuic acid, caftaric acid, glutathionyl caftaric acid (GRP), caffeic acid, p-coumaric acid, syringic acid, (-)-epicatechin, (+)-catechin and anthocyanins were identified in all four wine samples stored for thirty months (Table 4). Using one-way ANOVA, the differences in the phenolic profile for each type of wine were assessed by considering the closures as a factor. Regarding Merlot wines, GRP and syringic acid showed lower abundances in wines closed with the 'conventional' closure (technical cork $1+1$ ) than in those closed with the 'blend' corks, whereas (+)-cathechin and (-)-epicatechin were higher in the Merlot samples closed with technical $1+1$ closures.

Table 4. One-way ANOVA of the phenolic compounds found in each type of wine closed with both types of closure (closure effect). $\operatorname{Pr}>$ F indicates the $p$-value associated with the F statistic. The asterisk $\left.{ }^{*}\right)$ means significantly differences determined by Tukey's test $(\alpha<0.05)$. B and C: blend and conventional closure, respectively.

\begin{tabular}{|c|c|c|c|c|c|c|c|c|c|c|}
\hline Closure & $\begin{array}{l}\text { Gallic } \\
\text { Acid }\end{array}$ & $\begin{array}{l}\text { Protocatechuic } \\
\text { Acid }\end{array}$ & $\begin{array}{l}\text { Caftaric } \\
\text { Acid }\end{array}$ & $\begin{array}{l}\text { Glutathionyl } \\
\text { Caftaric } \\
\text { Acid }\end{array}$ & $\begin{array}{c}(+)- \\
\text { Catechin }\end{array}$ & $\begin{array}{l}\text { Caffeic } \\
\text { Acid }\end{array}$ & $\begin{array}{l}\text { Syringic } \\
\text { Acid }\end{array}$ & $\begin{array}{l}(-)- \\
\text { Epicatechin }\end{array}$ & $\begin{array}{c}p- \\
\text { Coumaric } \\
\text { Acid }\end{array}$ & Anthocyanins \\
\hline \multicolumn{11}{|c|}{ Merlot } \\
\hline $\mathrm{C}$ & 797902 & 19277 & 452477 & 64174 & 161170 & 109558 & 85709 & 92869 & 190434 & 86965 \\
\hline B & 775296 & 20349 & 442758 & 68093 & 143295 & 96415 & 91451 & 80817 & 179824 & 50231 \\
\hline $\operatorname{Pr}>\mathrm{F}$ & 0.065 & 0.063 & 0.308 & 0.001 * & 0.039 * & 0.193 & $0.045^{*}$ & 0.003 * & 0.371 & 0.157 \\
\hline \multicolumn{11}{|c|}{ Lagrein red } \\
\hline $\mathrm{C}$ & 529893 & 26416 & 534064 & 56755 & 116984 & 251583 & 179799 & 77357 & 349054 & 184361 \\
\hline B & 547415 & 29802 & 554889 & 61792 & 117628 & 244212 & 212296 & 100352 & 378623 & 133896 \\
\hline $\operatorname{Pr}>\mathrm{F}$ & 0.234 & $0.026^{*}$ & 0.459 & $0.026^{*}$ & 0.956 & 0.869 & 0.068 & 0.229 & 0.522 & 0.278 \\
\hline \multicolumn{11}{|c|}{ Lagrein rosé } \\
\hline $\mathrm{C}$ & 38832 & 9592 & 90022 & 24781 & 33893 & 20066 & 31342 & 6048 & 46235 & 16893 \\
\hline B & 38850 & 10169 & 88684 & 25115 & 37145 & 17456 & 32832 & 3878 & 41259 & 11602 \\
\hline $\operatorname{Pr}>\mathrm{F}$ & 0.966 & 0.637 & 0.463 & 0.668 & 0.078 & 0.051 & 0.102 & 0.040 * & 0.137 & 0.133 \\
\hline \multicolumn{11}{|c|}{ St. Magdalener } \\
\hline $\mathrm{C}$ & 144676 & 17699 & 471285 & 66081 & 173598 & 135204 & 83950 & 82501 & 245319 & 171631 \\
\hline B & 143419 & 19867 & 474859 & 76162 & 106769 & 78370 & 85387 & 60521 & 253125 & 77102 \\
\hline $\operatorname{Pr}>F$ & 0.848 & 0.082 & 0.748 & $0.005^{*}$ & 0.056 & $0.004^{*}$ & 0.807 & 0.001 * & 0.250 & $0.014^{*}$ \\
\hline
\end{tabular}

Concerning the red Lagrein wine, a significant difference was observed in two phenolic compounds: protocatechuic acid and GRP, with a low abundance in Lagrein closed with the 'conventional' closure (natural one-piece cork). In rosé Lagrein wine, (-)-epicatechin was higher in rosé closed with the 'conventional' closure (technical cork $1+1$ ) than in the wine closed with the 'blend' closure. Finally, the closures had an effect on the St. Magdalener wine, with a high abundance of caffeic acid, (-)-epicatechin and anthocyanins in the wines closed with the 'conventional' closure (agglomerated natural cork), while GRP was low in those closed with the 'conventional' closure.

The phenolic compounds that contributed the most to the red Lagrein wines sealed with both 'conventional' and 'blend' closures after 30 months of storage were p-coumaric and protocatechuic acids, anthocyanins, and caffeic and syringic acids, whereas gallic and caftaric acids, (-)-epicatechin, GRP, and (+)-catechin characterized the Merlot and St. Magdalener samples. Rosé wines had a low content of phenolic compounds due to their particular winemaking process (short maceration time), as has also been reported by Rossetti et al. [33]. 


\subsection{Sulfur Dioxide and Dissolved Oxygen Content}

One-way ANOVA was performed, considering the closure as a factor, on each wine to understand the influence of the closure type on dissolved oxygen, and free and total sulfites in each wine. The results presented in Table 5 show that only Lagrein rosé closed with the 'conventional' closure (technical cork 1+1) had a high content of free $\mathrm{SO}_{2}(21 \mathrm{mg} / \mathrm{L})$ compared to the 'blend' closure ( $12.5 \mathrm{mg} / \mathrm{L})$. On the other hand, no statistically significant difference was observed in free and total sulfites or dissolved oxygen for the Lagrein red wine, Merlot or St. Magdalener.

Table 5. One-way ANOVA for dissolved oxygen, free and total sulfur dioxide content of wines stored for 30 months and closed with blend closures. Pr $>$ F indicates the $p$-value associated with the F statistic. The asterisk $\left(^{*}\right)$ means significantly different groups as determined by Tukey's test $(\alpha<0.01)$. $\mathrm{B}$ and $\mathrm{C}$ : blend and conventional closure, respectively.

\begin{tabular}{|c|c|c|c|}
\hline Closures & $\begin{array}{l}\text { Total Sulfur Dioxide } \\
(\mathrm{mg} / \mathrm{L})\end{array}$ & $\begin{array}{l}\text { Free Sulfur Dioxide } \\
(\mathrm{mg} / \mathrm{L})\end{array}$ & $\begin{array}{c}\text { Oxygen Content } \\
(\mathrm{mg} / \mathrm{L})\end{array}$ \\
\hline \multicolumn{4}{|c|}{ Merlot } \\
\hline $\mathrm{C}$ & 38.50 & 26.00 & 1.85 \\
\hline B & 26.50 & 20.00 & 1.05 \\
\hline $\operatorname{Pr}>F$ & 0.077 & 0.238 & 0.064 \\
\hline \multicolumn{4}{|c|}{ Lagrein red } \\
\hline $\mathrm{C}$ & 37.00 & 22.50 & 0.15 \\
\hline B & 28.50 & 25.00 & 0.10 \\
\hline $\operatorname{Pr}>F$ & 0.054 & 0.649 & 0.423 \\
\hline \multicolumn{4}{|c|}{ Lagrein rosé } \\
\hline $\mathrm{C}$ & 96.00 & 21.00 & 2.00 \\
\hline $\mathrm{B}$ & 83.00 & 12.50 & 2.00 \\
\hline $\operatorname{Pr}>F$ & 0.049 & $0.003 *$ & 1.000 \\
\hline \multicolumn{4}{|c|}{ St. Magdalener } \\
\hline $\mathrm{C}$ & 36.00 & 16.00 & 0.25 \\
\hline B & 19.00 & 11.50 & 0.05 \\
\hline $\operatorname{Pr}>F$ & 0.033 & 0.057 & 0.106 \\
\hline
\end{tabular}

\subsection{Correlations between Sulfur Dioxide, Dissolved Oxygen and Volatile and Phenolic Profiles}

A PCA was applied to understand the influence of $\mathrm{SO}_{2}$ and dissolved $\mathrm{O}_{2}$ content on the wine quality. Figure $1 \mathrm{~A}$ shows that total $\mathrm{SO}_{2}$ and $\mathrm{O}_{2}$ contributed together, along with some identified volatile compounds such as butanediol (3), 1-hexanol (8), ethyl hexanoate (13), ethyl octanoate (25), and ethyl decanoate (28), to characterize the Lagrein rosé wines closed with both closures.

Another PCA plot (Figure 1B) was obtained by excluding the rosé Lagrein wines, as they had a lower phenolic content than the other three wines, with the aim of better observing the relationship of $\mathrm{SO}_{2}$ and $\mathrm{O}_{2}$ with the volatile and phenolic profiles of the red wines. The PCA graph showed that octanoic acid (22) and diethyl succinate (23) showed a stronger relationship with the $\mathrm{O}_{2}$ content and mainly characterized the Merlot samples. On the other hand, the content of free and total $\mathrm{SO}_{2}$ were directly correlated with (-)-epicatechin. 

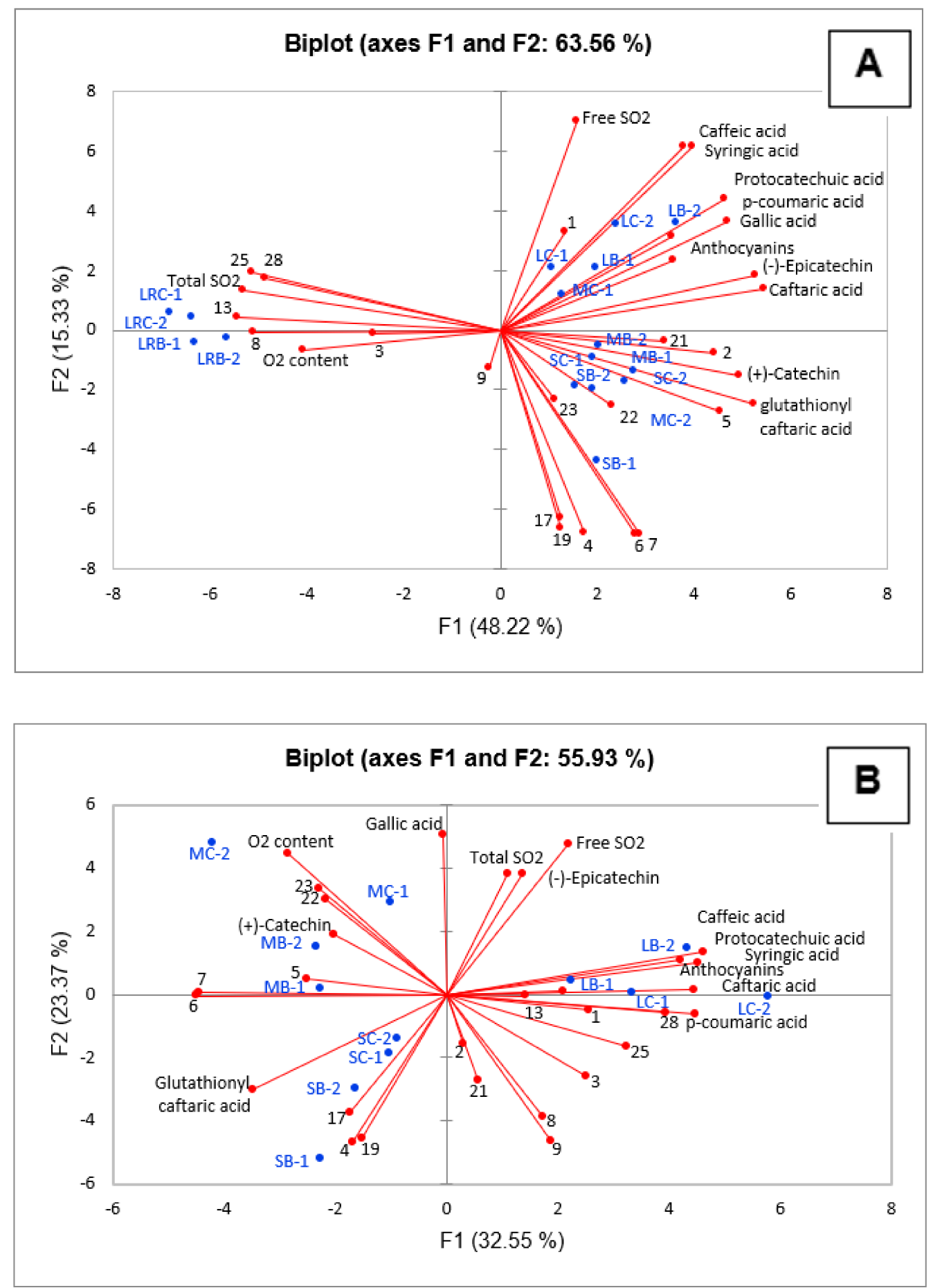

Figure 1. PCA bi-plot of volatile and non-volatile compounds, $\mathrm{SO}_{2}$ and dissolved $\mathrm{O}_{2}$ contents determined in the wines after 30 months of storage. (A) F1 vs F2, Principal Components of three red and one rosé wine; (B): F1 vs F2; Principal Components of three red wines excluding the rosé wine. LRB, LRC: Lagrein rosé closed with 'blend' or 'conventional' closure, respectively; SB, SC: same for St. Magdalener; LB, LC: same for Lagrein red; MB, MC: same for Merlot. Volatile compounds are represented by numbers, as listed in Table 2 . Values were not averaged for experimental replicates (-1: bottle 1, -2: bottle 2$)$.

Two aspects concerning both PCA plots are noteworthy: in both models, (i) glutathionyl caftaric acid was inversely correlated with total $\mathrm{SO}_{2}$ content and (ii) three fermen- 
tation volatile compounds, including ethyl butanoate (4), isoamyl lactate (17), and octanol (19), were inversely correlated with both free and total $\mathrm{SO}_{2}$.

\subsection{Sensory Evaluation}

The triangle test (Table 6) performed on the four wines at 30 months of storage only showed a significant difference for the St. Magdalener and Lagrein rosé wines. Both wines closed with 'conventional' closures were different from the 'blend closure'. On the other hand, no statistical difference was found between the Merlot wines closed with 'conventional' and 'blend' closures, or for the Lagrein red wines.

Table 6. Triangle test. ns: not significant; ${ }^{*}$ significant $(\alpha<0.05)$.

\begin{tabular}{cccc}
\hline Merlot & Lagrein Red & Lagrein Rosé & St. Magdalener \\
\hline $\mathrm{ns}$ & $\mathrm{ns}$ & $*$ & $*$ \\
\hline
\end{tabular}

\section{Discussion}

For the first time, South Tyrolean wines (three red and one rosé) stored for thirty months in bottles and sealed with different types of closures were studied in terms of changes in volatile and phenolic compounds and their content of free and total sulfites and dissolved oxygen.

The concept of this work was not the absolute quantification of volatile compounds and phenols in these red and rosé wines, but to investigate if storage with different closures could have an impact on the chemical composition of wines. For this purpose, an absolute quantification is not necessary; in fact, ANOVA and multivariate statistical models such as PCA are based on relative abundances (and their variance), and not on an absolute quantification.

One-way ANOVA was considered the best approach for this study, as the "blend" closure was compared for each wine with a conventional closure analyzed after a prolonged storage of the wines in bottles.

The closure type (blend $v s$ conventional) did not significantly affect $(\alpha<0.01)$ free or total $\mathrm{SO}_{2}$ or the dissolved oxygen content of any of the wines, with the exception of the free $\mathrm{SO}_{2}$ content of Lagrein rosé. This means that, in general terms, all the different types of corks examined mostly ensured good hermeticity during the first 30 months of storage in bottles. In particular, this is important for the winemaker, whose first concern is to maintain the levels of free (and total) sulfites present at bottling, in order to ensure the protection of the wine from oxidation and abnormal fermentations. In the case examined, it was not found that there was a systematic difference in the content of free sulfites in any of the wines according to the type of cork used.

As the differences in free and total sulfite and dissolved oxygen content among the corks used were almost never significant, it is not straightforward to describe the mechanisms that can be predicted from the chemical data here-particularly the influence of oxygen transfer through the cork on various phenolic compounds, sulfur dioxide, and volatiles.

After thirty months of storage, without taking into account the effect of the type of closure, many volatile compounds with a number of carbon atoms from 7 to 18, including long-chain ethyl esters, disappeared, while some esters appeared-probably due to the condensation of higher alcohols (e.g., isoamyl alcohol) with organic acids formed after fermentation. In addition, some higher alcohols and C7 and C8 aldehydes disappearedprobably due to oxidation.

A significant difference in the volatile profiles was observed only in Lagrein red wine closed with the 'conventional' closure, which showed a higher content of 2, 3-butanediol and ethyl octanoate compared to the 'blend' cork after 30 months. Octanoic acid and diethyl succinate (fermentative volatile compounds) contributed to distinguishing the Merlot samples, which showed the highest dissolved oxygen content. Regarding the 
differences in phenolic content induced by the closure used, GRP and (-)-epicatechin were the compounds that most consistently changed in the wines. In general, the cinnamic acids, flavanols, and phenolic acids were the most affected by the closure, while anthocyanins differentiated by the closure used only in the St. Magdalener wines. In our case, when the differences were indeed significant, conventional closure-closed wines generally showed higher relative abundances of epicatechin in three of the four wines and lower relative abundances of GRP (in all the three red wines) and other phenolic acids, such as syringic and protocatechuic acids. Finally, anthocyanins and caffeic acid were significantly higher in the St. Magdalener wines closed with conventional closures.

Xing et al. [54] have also reported that storage time has a major effect on the maturation of phenolic compounds, with considerable loss of most phenolic compounds after 18 months in a bottle. Similar results were observed in the present study (30-month storage), where most phenols decreased compared to the results obtained up to 12 months [33]. Xing et al. [54] also demonstrated that there was no strong correlation between the phenolic variation and the dissolved oxygen content. Silva et al. [55] also performed a similar study evaluating the effect of synthetic, agglomerated and natural one-piece cork closures on white, red and rosé wines. Their results showed that the usage of synthetic closures helped to increase the shelf-life of wines within a period of 1-2 years after bottling. Their results are in line with the present research, showing that the different type of closures (a natural one-piece cork, a blend of natural cork microgranules, a technical cork $1+1$ and an agglomerated natural cork) used to close the four wines (Merlot, Lagrein red and rosé and St. Magdalener) for up to 30 months of storage did not considerably affect the final quality of the wines.

Although the St. Magdalener and Lagrein rosé wines closed with 'conventional' or 'blend' closures could be discriminated using the triangle test, these differences appeared to be relative, as they did not include all the four wines systematically, but rather appeared to be due to compositional differences in the content of the individual wines. The triangle test was chosen for its ability in aiding the investigation of possible sensory differences that may be present in the wines depending on the type of closure, although the reasons for and the degree of these differences cannot be directly explained with this test.

Author Contributions: Conceptualization, E.B. and E.L.; methodology, E.L. and A.D.d.M.; software, P.F.T.B.; validation, E.B., E.L., and P.F.T.B.; formal analysis, P.F.T.B.; V.M.; A.D.d.M.; E.L.; investigation, P.F.T.B.; E.L.; E.B.; F.R.; V.M.; A.D.d.M.; resources, E.B.; data curation, P.F.T.B.; E.L.; E.B.; V.M.; A.D.d.M.; writing—original draft preparation, P.F.T.B.; writing-review and editing, P.F.T.B.; E.B.; E.L.; V.M.; F.R.; A.D.d.M.; visualization, E.B.; supervision, E.B.; E.L.; project administration, E.B.; funding acquisition, E.B. All authors have read and agreed to the published version of the manuscript.

Funding: This research was funded by the Oenolab Start-up Project (TN2810) and the WineID Project (TN201A) of the Free University of Bozen-Bolzano. This work was supported by the Open Access Publishing Fund of the Free University of Bozen-Bolzano.

Institutional Review Board Statement: Not applicable.

Informed Consent Statement: Informed consent was obtained from all subjects involved in the triangle test.

Data Availability Statement: The data presented in this study are available on request from the corresponding author.

Acknowledgments: Kellerei Bozen (Bolzano, Italy) is kindly acknowledged for providing the wines. We also thank Supercap srl (Mombaroccio, Italy) for providing the closures (a sanitized micro-granule blend of natural cork with polymers without the addition of glue to the mix) used in this work and for the determination of the oxygen content.

Conflicts of Interest: The authors declare no conflict of interest. The funders had no role in the design of the study; in the collection, analyses, or interpretation of data; in the writing of the manuscript, or in the decision to publish the results. 


\section{References}

1. Ribéreau-Gayon, P.; Glories, Y.; Maujean, A. Handbook of Enology, Volume 2: The Chemistry of Wine-Stabilization and Treatments (Volume 2); Dubourdieu, D., Ed.; John Wiley \& Sons: Chichester, UK, 2006.

2. Hernanz, D.; Gallo, V.; Ángeles, F.R.; Meléndez-Martínez, A.J.; González-Miret, M.L.; Heredia, F.J. Effect of storage on the phenolic content, volatile composition and colour of white wines from the varieties Zalema and Colombard. Food Chem. 2009, 113, 530-537. [CrossRef]

3. Boulton, R.B.; Singleton, V.L.; Bisson, L.F.; Kunkee, R.E. Principles and Practices of Winemaking; Springer Science \& Business Media: New York, NY, USA, 2013.

4. Varea, S.; García-Vallejo, M.; Cadahia, E.; de Simón, F.B. Polyphenols susceptible to migrate from cork stoppers to wine. Eur. Food Res. Technol. 2001, 213, 56-61. [CrossRef]

5. Skouroumounis, G.; Kwiatkowski, M.; Francis, I.; Oakey, H.; Capone, D.; Duncan, B.; Sefton, M.; Waters, E. The impact of closure type and storage conditions on the composition, colour and flavour properties of a Riesling and a wooded Chardonnay wine during five years' storage. Aust. J. Grape Wine Res. 2005, 11, 369-377. [CrossRef]

6. González-Adrados, J.R.; González-Hernández, F.; De Ceca, J.L.G.; Cáceres-Esteban, M.J.; García-Vallejo, M.C. Cork-wine interaction studies: Liquid absorption and non-volatile compound migration. OENO One 2008, 42, 163-166. [CrossRef]

7. Azevedo, J.; Fernandes, I.; Lopes, P.; Roseira, I.; Cabral, M.; Mateus, N.; Freitas, V. Migration of phenolic compounds from different cork stoppers to wine model solutions: Antioxidant and biological relevance. Eur. Food Res. Technol. 2014, 239, 951-960. [CrossRef]

8. Waters, E.; Peng, Z.; Pocock, K.; Williams, P. The role of corks in oxidative spoilage of white wines. Aust. J. Grape Wine Res. 1996, 2, 191-197. [CrossRef]

9. Mas, A.; Puig, J.; Lladoa, N.; Zamora, F. Sealing and Storage Position Effects on Wine Evolution. J. Food Sci. 2002, 67, 1374-1378. [CrossRef]

10. Lopes, P.; Saucier, C.; Teissedre, A.P.-L.; Glories, Y. Impact of Storage Position on Oxygen Ingress through Different Closures into Wine Bottles. J. Agric. Food Chem. 2006, 54, 6741-6746. [CrossRef]

11. Karbowiak, T.; Gougeon, R.; Alinc, J.-B.; Brachais, L.; Debeaufort, F.; Voilley, A.; Chassagne, D. Wine Oxidation and the Role of Cork. Crit. Rev. Food Sci. Nutr. 2009, 50, 20-52. [CrossRef]

12. Silva, M.A.; Julien, M.; Jourdes, M.; Teissedre, P.-L. Impact of closures on wine post-bottling development: A review. Eur. Food Res. Technol. 2011, 233, 905-914. [CrossRef]

13. Ugliano, M. Oxygen Contribution to Wine Aroma Evolution during Bottle Aging. J. Agric. Food Chem. 2013, 61, 6125-6136. [CrossRef] [PubMed]

14. Gambuti, A.; Siani, T.; Picariello, L.; Rinaldi, A.; Lisanti, M.T.; Ugliano, M.; Dieval, J.B.; Moio, L. Oxygen exposure of tannins-rich red wines during bottle aging. Influence on phenolics and color, astringency markers and sensory attributes. Eur. Food Res. Technol. 2017, 243, 669-680. [CrossRef]

15. Marais, J.; Pool, H.J. Effect of storage time and temperature on the volatile composition and quality of dry white table wines. Vitis 1980, 19, 151-164.

16. Simpson, R.F.; Miller, G.C. Aroma composition of aged Riesling wine. Vitis 1983, 22, 2251-2263.

17. Ugliano, M.; Kwiatkowski, M.J.; Travis, B.; Francis, I.L.; Waters, E.J.; Herderich, M.J.; Pretorius, I.S. Post-bottling management of oxygen to reduce off-flavour formation and optimize wine style. Aust. N. Zealand Wine Ind. J. 2009, 24, 24-28.

18. Hart, A.; Kleinig, A. The role of oxygen in the aging of bottled wine. Aust. N. Zealand Wine Industry J. 2005, $20,46-50$.

19. Lopes, P.; Silva, M.A.; Pons, A.; Tominaga, T.; Lavigne, V.; Saucier, C.; Dubourdieu, D. Impact of oxygen dis-solved at bottling and transmitted through closures on the composition and sensory properties of a Sauvignon blanc wine during bottle storage. J. Agric. Food Chem. 2009, 57, 10261-10270. [CrossRef]

20. Escudero, A.; Asensio, E.; Cacho, J.; Ferreira, V. Sensory and chemical changes of young white wines stored under oxygen. An assessment of the role played by aldehydes and some other important odorants. Food Chem. 2002, 77, 325-331. [CrossRef]

21. Mozzon, M.; Savini, S.; Boselli, E.; Thorngate, J.H. The herbaceous character of wines. Ital. J. Food Sci. 2016, $28,190$.

22. Skouroumounis, G.; Kwiatkowski, M.; Francis, I.; Oakey, H.; Capone, D.; Peng, Z.; Duncan, B.; Sefton, M.; Waters, E. The influence of ascorbic acid on the composition, colour and flavour properties of a Riesling and a wooded Chardonnay wine during five years' storage. Aust. J. Grape Wine Res. 2005, 11, 355-368. [CrossRef]

23. Arapitsas, P.; Dalledonne, S.; Scholz, M.; Catapano, A.; Carlin, S.; Mattivi, F. White wine light-strike fault: A comparison between flint and green glass bottles under the typical supermarket conditions. Food Packag. Shelf Life 2020, 24, 100492. [CrossRef]

24. Fracassetti, D.; Gabrielli, M.; Encinas, J.; Manara, M.; Pellegrino, L.M.; Tirelli, A. Approaches to prevent the light-struck taste in white wine. Aust. J. Grape Wine Res. 2017, 23, 329-333. [CrossRef]

25. Benítez, P.; Castro, R.; Natera, R.; Barroso, C.G. Changes in the polyphenolic and volatile content of "Fino" Sherry wine exposed to high temperature and ultraviolet and visible radiation. Eur. Food Res. Technol. 2006, 222, 302-309. [CrossRef]

26. Betnga, P.F.T.; Longo, E.; Poggesi, S.; Boselli, E. Effects of transport conditions on the stability and sensory quality of wines. OENO One 2021, 55, 4524. [CrossRef]

27. Chung, H.-J.; Son, J.-H.; Park, E.-Y.; Kim, E.-J.; Lim, S.-T. Effect of vibration and storage on some physico-chemical properties of a commercial red wine. J. Food Compos. Anal. 2008, 21, 655-659. [CrossRef] 
28. Chatonnet, P.; Labadie, D.; Gubbiotti, M.-C. Comparative study of different types of corkage. Rev. Oeno-Logues Tech. Vitivinic. Oenologiques 2000, 95, 7-13.

29. Godden, P.; Francis, L.; Field, J.; Gishen, M.; Coulter, A.; Valente, P.; Høj, P.; Robinson, E. Wine bottle closures: Physical characteristics and effect on composition and sensory properties of a Semillon wine 1. Performance up to 20 months post-bottling. Aust. J. Grape Wine Res. 2001, 7, 64-105. [CrossRef]

30. Godden, P.; Lattey, K.; Francis, L.; Gishen, M.; Cowey, G.; Holdstock, M.; Capone, D. Towards offering wine to the consumer in optimal condition-The wine, the closures and other packaging variables. Wine Ind. J. 2005, 20, 20-30.

31. Kwiatkowski, M.J.; Skouroumounis, G.K.; Lattey, K.A.; Waters, E.J. The impact of closures, including screw cap with three different headspace volumes, on the composition, colour and sensory properties of a Cabernet Sauvignon wine during two years' storage. Aust. J. Grape Wine Res. 2007, 13, 81-94. [CrossRef]

32. Liu, N.; Song, Y.-Y.; Dang, G.-F.; Ye, D.-Q.; Gong, X.; Liu, Y.-L. Effect of Wine Closures on the Aroma Properties of Chardonnay Wines after Four Years of Storage. South Afr. J. Enol. Vitic. 2015, 36, 296-303. Available online: http://www.scielo.org.za/pdf/ sajev/v36n3/07.pdf (accessed on 24 November 2021). [CrossRef]

33. Rossetti, F.; Jouin, A.; Jourdes, M.; Teissedre, P.-L.; Foligni, R.; Longo, E.; Boselli, E. Impact of Different Stoppers on the Composition of Red and Rosé Lagrein, Schiava (Vernatsch) and Merlot Wines Stored in Bottle. Molecules 2020, 25, 4276. [CrossRef] [PubMed]

34. Longo, E.; Rossetti, F.; Jouin, A.; Teissedre, P.-L.; Jourdes, M.; Boselli, E. Distribution of crown hexameric procyanidin and its tetrameric and pentameric congeners in red and white wines. Food Chem. 2019, 299, 125125. [CrossRef] [PubMed]

35. De Matos, A.D.; Longo, E.; Chiotti, D.; Pedri, U.; Eisenstecken, D.; Sanoll, C.; Robatscher, P.; Boselli, E. Pinot Blanc: Impact of the Winemaking Variables on the Evolution of the Phenolic, Volatile and Sensory Profiles. Foods 2020, 9, 499. [CrossRef]

36. Klesk, K.; Qian, M. Aroma Extract Dilution Analysis of Cv. Marion (Rubus spp. hyb) and Cv. Evergreen (R. laciniatus L.) Blackberries. J. Agric. Food Chem. 2003, 51, 3436-3441. [CrossRef] [PubMed]

37. Xie, J.; Sun, B.; Zheng, F.; Wang, S. Volatile flavor constituents in roasted pork of Mini-pig. Food Chem. 2008, 109, 506-514. [CrossRef]

38. Krist, S.; Stuebiger, G.; Unterweger, H.; Bandion, F.; Buchbauer, G. Analysis of Volatile Compounds and Triglycerides of Seed Oils Extracted from Different Poppy Varieties (Papaver somniferum L.). J. Agric. Food Chem. 2005, 53, 8310-8316. [CrossRef]

39. Iraqi, R.; Vermeulen, C.; Benzekri, A.; Bouseta, A.; Collin, S. Screening for Key Odorants in Moroccan Green Olives by Gas Chromatography-Olfactometry/Aroma Extract Dilution Analysis. J. Agric. Food Chem. 2005, 53, 1179-1184. [CrossRef] [PubMed]

40. Kim, T.H.; Shin, J.H.; Yu, E.J.; Kim, Y.-S.; Lee, H.J.; Kim, T.H. Characteristics of aroma-active compounds in the pectin-elicited suspension culture of Zanthoxylum piperitum (prickly ash). Biotechnol. Lett. 2002, 24, 551-556. [CrossRef]

41. Beaulieu, J.C.; Grimm, C.C. Identification of Volatile Compounds in Cantaloupe at Various Developmental Stages Using Solid Phase Microextraction. J. Agric. Food Chem. 2001, 49, 1345-1352. [CrossRef]

42. Sampaio, T.S.; Nogueira, P.C.L. Volatile components of mangaba fruit (Hancornia speciosa Gomes) at three stages of maturity. Food Chem. 2006, 95, 606-610. [CrossRef]

43. NIST Chemistry WebBook, SRD 69. Available online: https://webbook.nist.gov/cgi/inchi/InChI\%3D1S/C8H16O3/c1-6(2)4-511-8(10)7(3)9/h6-7\%2C9H\%2C4-5H2\%2C1-3H3 (accessed on 24 November 2021).

44. Xu, X.; Stee, L.L.P.; Williams, J.; Beens, J.; Adahchour, M.; Vreuls, R.J.J.; Brinkman, U.A.; Lelieveld, J. Comprehensive twodimensional gas chromatography $(\mathrm{GC} \times \mathrm{GC})$ measurements of volatile organic compounds in the atmosphere. Atmos. Chem. Phys. Discuss. 2003, 3, 665-682. [CrossRef]

45. Schirack, A.V.; Drake, M.A.; Sanders, T.H.; Sandeep, K.P. Characterization of aroma-active compounds in microwave blanched peanuts. J. Food Sci. 2006, 71, C513-C520. [CrossRef]

46. Passos, X.S.; Castro, A.C.M.; Pires, J.S.; Garcia, A.C.F.; Campos, F.C.; Fernandes, O.F.L.; de Paula, J.R.; Ferreira, H.D.; Santos, S.C.; Ferri, P.H.; et al. Composition and Antifungal Activity of the Essential Oils of Caryocar brasiliensis. Pharm. Biol. 2003, 41, 319-324. [CrossRef]

47. Su, Y.C.; Ho, C.L.; Wang, E.I.; Chang, S.T. Antifungal activities and chemical compositions of essential oils from leaves of four eucalypts. Taiwan J. For. Sci. 2006, 21, 49-61.

48. Flamini, G.; Cioni, P.L.; Morelli, I. Volatiles from Leaves, Fruits, and Virgin Oil from Olea europaea Cv. Olivastra Seggianese from Italy. J. Agric. Food Chem. 2003, 51, 1382-1386. [CrossRef] [PubMed]

49. Fan, G.; Lu, W.; Yao, X.; Zhang, Y.; Wang, K.; Pan, S. Effect of fermentation on free and bound volatile compounds of orange juice. Flavour Fragr. J. 2009, 24, 219-225. [CrossRef]

50. Boulanger, R.; Crouzet, J. Free and bound flavour components of Amazonian fruits: 3-glycosidically bound components of cupuacu. Food Chem. 2000, 70, 463-470. [CrossRef]

51. Fariña, L.; Villar, V.; Ares, G.; Carrau, F.; Dellacassa, E.; Boido, E. Volatile composition and aroma profile of Uruguayan Tannat wines. Food Res. Int. 2015, 69, 244-255. [CrossRef]

52. Betnga, P.F.T.; de Matos, A.D.; Longo, E.; Boselli, E. Impact of closure material on the chemical and sensory profiles of grappa during storage in bottle. LWT 2020, 133, 110014. [CrossRef] 
53. Bowen, A.J.; Reynolds, A.G. Odor Potency of Aroma Compounds in Riesling and Vidal blanc Table Wines and Icewines by Gas Chromatography-Olfactometry-Mass Spectrometry. J. Agric. Food Chem. 2012, 60, 2874-2883. [CrossRef]

54. Xing, R.R.; Liu, D.; Li, Z.; Tian, Y.; Zhang, X.X.; Li, J.M.; Pan, Q.H. Impact of different types of closures on sensorial and phenolic characteristics evolution during a bottle storage time of a white wine from Chardonnay grape variety. J. Food Sci. Technol. 2016, 53, 4043-4055. [CrossRef] [PubMed]

55. Silva, A.; Lambri, M.; De Faveri, M.D. Evaluation of the performances of synthetic and cork stoppers up to 24 months post-bottling. Eur. Food Res. Technol. 2003, 216, 529-534. [CrossRef] 